\title{
Strain-hardening properties of the high-entropy alloy MoNbTaTiVZr processed by high-pressure torsion
}

Received: 24 August 2021 / Accepted: 29 October 2021 / Published online: 25 November 2021

(C) The Author(s) 2021

\begin{abstract}
An equiatomic MoNbTaTiVZr refractory high-entropy alloy (HEA) produced by arc melting was processed by high-pressure torsion (HPT) at room temperature. Thermodynamic calculations and experimental results indicated a dual-phase microstructure composed of about $85 \%$ BCC Zr-depleted and $15 \%$ BCC Zr-rich phase in the as-cast condition. HPT causes grain refinement and an increase in dislocation density without the formation of new phases. After four revolutions, the Zr-depleted phase was hardened to $\sim 540 \mathrm{HV}$, while the $\mathrm{Zr}$-rich phase exhibited softening with a decrease in hardness to $\sim 480 \mathrm{HV}$. The occurrence of a vortex-like microstructure and the analysis of elemental concentrations indicated a shear-induced mechanical homogenization, which was supposed to be the cause of the observed softening.
\end{abstract}

Keywords High-entropy alloy - Severe plastic deformation - CALPHAD · High-energy X-ray diffraction · Mechanical homogenization

\section{Introduction}

In recent years, high-entropy alloys (HEAs) have received much attention from researchers worldwide due to their outstanding properties [1-4]. Unlike conventional alloys which usually contain only one principle element, HEAs consist of at least five major elements in equiatomic or near-equiatomic proportions [5]. Favored by the high configurational entropy resulting from the mixing of multiple elements, HEAs are expected to form single-phase disordered solid solutions instead of intermetallic compounds [5]. In practice, heterogeneous phase compositions including the combination of solid solution with compounds can also be found in HEAs, which exhibit enhanced mechanical properties compared to single-phase solid solutions [6,7]. HEAs composed

Communicated by Andreas Öchsner.

The original online version of this article was revised: the figure caption of figure 1 and 2 has been swapped. This has been corrected in original article.

C. Duan $(\varangle) \cdot$ M. Reiberg $\cdot$ E. Werner

Institute of Materials Science and Mechanics of Materials, Technical University of Munich, Boltzmannstr. 15, 85748 Garching, Germany

E-mail: chuyi.duan@tum.de

P. Kutlesa $\cdot$ R. Pippan

Erich Schmid Institute of Materials Science, Austrian Academy of Sciences, Jahnstr. 12, 8700 Leoben, Austria

X. Li

Heinz Maier-Leibnitz Zentrum (MLZ), Technical University of Munich, Lichtenbergstr. 1, 85748 Garching, Germany 
of refractory elements (also referred to as refractory HEAs) show superior high temperature properties and can be of great interest for elevated temperature applications [1,8]. However, refractory HEAs often possess a limited ductility at low temperature $[1,9,10]$. In order to study their behavior during plastic deformation, particularly the strain-hardening properties, special experimental equipment and methods have to be employed.

As a severe plastic deformation (SPD) technique, high-pressure torsion (HPT) is a well-established method to fabricate nano-crystalline material states and is one of the possibilities to induce very high plastic strains even in materials with poor workability [11,12]. Benefiting from this advantage, HPT can be utilized to process refractory HEAs. Apart from nanocrystallization and strain-hardening studies, HPT was also applied to investigate the mixing of two dissimilar materials during mechanical alloying [13-15]. By plastic shearing of materials, HPT can impose large amounts of crystal defects, e.g. dislocations, grain boundaries and nonequilibrium vacancies, which can accelerate atomic diffusion and result in a mechanical homogenization [16-18].

The goal of this work is to investigate the strain-hardening properties of a dual-phase refractory HEA and the mixing behavior of the two phases during HPT. Although several recent studies have been reported on HPT-processed single-phase FCC HEAs [19-22] as well as single-phase BCC refractory HEAs [23,24], the strain-hardening behavior of dual-phase refractory HEAs processed by HPT has only been studied recently $[25,26]$.

In this study, the microstructure and hardness evolution of the as-cast condition and of HPT-samples after 0 (as-compressed), 1/4, 2 and 4 revolutions were analyzed employing scanning electron microscopy, X-ray diffraction (XRD) and ultra-microhardness testing (UMHT). A comprehensive evaluation of the relationship between hardness and elemental concentration was conducted.

\section{Experimental procedures}

\subsection{Sample processing}

An HEA with the equiatomic composition of MoNbTaTiVZr was produced by mixing and arc melting pure elements (purity > $99.5 \mathrm{wt} . \%$ ) under high-purity argon atmosphere in a water-cooled copper crucible. To avoid the contamination by oxygen, a Ti-getter was used, and the chamber was evacuated and ventilated with argon for at least five times prior to melting. The ingot was flipped over and remelted 10 times, and during the last step kept in liquid state for 2 minutes. The button-shaped ingot with dimensions of about $14 \times 14 \times 8 \mathrm{~mm}^{3}$ was machined to a cylindrical specimen with a diameter of $6 \mathrm{~mm}$ using electric discharge machining (EDM). The cylindrical specimen was ground with 2000-grit $\mathrm{SiC}$ abrasive paper to remove the oxidation layer on its surface. Disk-shaped specimens with a diameter of $6 \mathrm{~mm}$ and a thickness of $\sim 0.8 \mathrm{~mm}$ were sliced from the cylindrical specimen using a wire diamond saw (Fig. 1c). These specimens are referred to as initial (as-cast) condition in the following.

HPT experiments were conducted at the Erich Schmid Institute of Materials Science, Leoben, Austria. The disks were processed by a conventional quasi-constrained HPT facility with Bridgman-anvils ( $6 \mathrm{~mm}$ diameter, $0.6 \mathrm{~mm}$ total depth). Details of this experimental setup can be found in [27-29]. In order to avoid slipping, the samples were sand blasted to increase the friction between the anvils and the disk. The disks were subjected to $0,1 / 4,2$ and 4 revolutions under a hydrostatic pressure of $7 \mathrm{GPa}$ with a rate of 0.2 revolutions per minute (RPM) at room temperature (RT). The disk with 0 revolutions was merely compressed by the anvils without introducing shear strains.

\subsection{Characterization}

Thermodynamic equilibrium calculations were conducted with the software ThermoCalc (TCHEA database) using the CALPHAD (CALculation of PHAse Diagrams) method to predict the phase stability and the elemental concentration in the phases present.

The phase composition of the as-cast sample was examined experimentally using synchrotron high-energy X-ray diffraction (HEXRD) at the High Energy Materials Science (HEMS) beamline of the Helmholtz-Zentrum Geesthacht at PETRA III at DESY in Hamburg, Germany. The beam had a photon energy of $100 \mathrm{keV}$, a spot size of $0.5 \times 0.5 \mathrm{~mm}^{2}$, and a wavelength of $0.124 \AA$. The diffracted beam was analyzed using a PerkinElmer XRD 1622 flat panel detector with a distance to the sample of about $1600 \mathrm{~mm}$. The results were averaged 


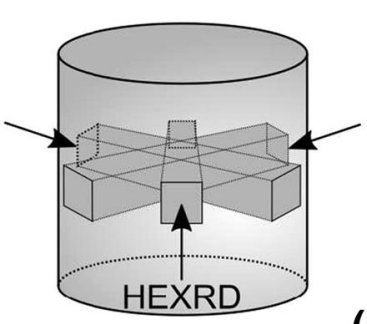
incident beam

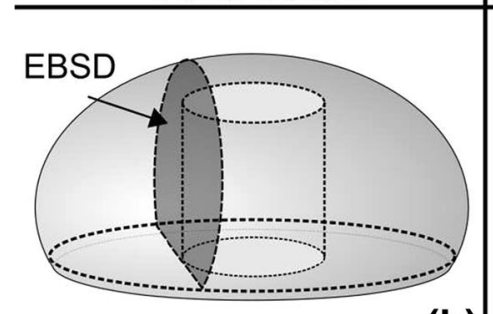

(b)

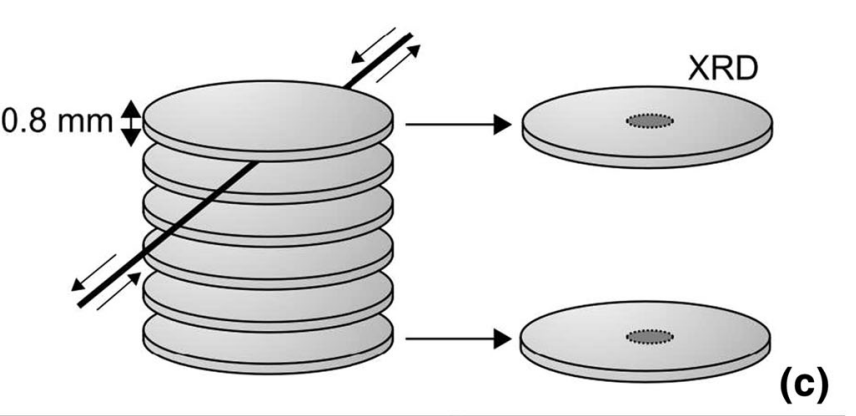

(c)

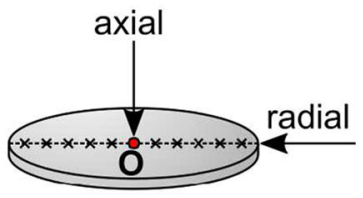

(e)

Fig. 1 Sketch of experimental methods: (a) high-energy X-ray diffraction (HEXRD) for the as-cast cylindrical specimen; (b) process of slicing using a wire diamond saw and the position selected for XRD in as-cast disks; (c) electron backscatter diffraction (EBSD) for specimen in the as-cast condition; (d) positions on HPT-processed disks for XRD and (e) positions for hardness and elemental concentration measurements

from three measurements along the radial direction with an angular distance of $120^{\circ}$ (Fig. 1a) and were calibrated using an $\mathrm{LaB}_{6}$ powder standard. Rietveld full-profile analysis was carried out with the software MAUD [30].

For the following experiments, the samples were firstly embedded, then ground with 320- to 1000-grit SiC abrasive papers and polished with $3 \mu \mathrm{m}$ and $1 \mu \mathrm{m}$ diamond suspensions and finally OPS (oxide polishing suspension) with a grain size of $0.25 \mu \mathrm{m}$ and $\mathrm{pH}$ value of 9-10.

The phases present in the disks both before and after HPT were examined by XRD using a Bruker D8 Xenia diffractometer operating with $\mathrm{Cu}-\mathrm{K} \alpha$-radiation at $40 \mathrm{kV}$ and $15 \mathrm{~mA}$. The spot was circular and had a diameter of $0.5 \mathrm{~mm}$. Before HPT, the diffractograms collected from the centers of the top and bottom disks were compared to study the homogeneity of the cylindrical specimen in axial direction. After HPT, 8 positions located on a circle with a radius of $2 \mathrm{~mm}$ on the disks were selected for measurement (Fig. 1d). The results were averaged from these individual measurements. Microstructure observations and chemical composition analysis were conducted using a scanning electron microscope (SEM) JEOL JSM6490 equipped with an Oxford energy-dispersive X-ray spectroscopy (EDS) detector. The grain structure and phase distribution were studied using electron backscattered diffraction (EBSD) in a field emission SEM (JEOL JSM-7600F) equipped with an Oxford EBSD detector. EBSD examinations on the sample in the ascast condition were performed on the remaining material of the same ingot used for HPT disks (Fig. 1b). Investigation on HPT-processed disks was performed at radii of 1,2 and $2.5 \mathrm{~mm}$ with different magnifications. Depending on the grain size, the step size (pixel distance at a selected magnification) was set to 1 (as-cast), 0.2376 ( 0 and $1 / 4$ revolution) and $0.0393 \mu \mathrm{m}$ ( 2 and 4 revolutions), respectively. A combined EBSD-EDS detection system was used to distinguish the two BCC phases exhibiting little difference in lattice constants.

To investigate the hardening properties during HPT, ultra-microhardness measurements were conducted at RT using a UMHT-1 tester (Kammrath \& Weiss GmbH, Schwerte, Germany) built into the SEM JEOL JSM6490 applying a force of $20 \mathrm{mN}$, a dwell time of $10 \mathrm{~s}$, and a loading velocity of $0.25 \mathrm{mN} / \mathrm{s}$. The hardness was measured at the center and at 10 positions with a distance of $0.5 \mathrm{~mm}$ from the center along the diameter of the HPT disks (Fig. 1e). The length of the diagonals of the indents were measured using the software ImageJ [31]. At each position, the hardness value of the two phases was the average from five indents. 


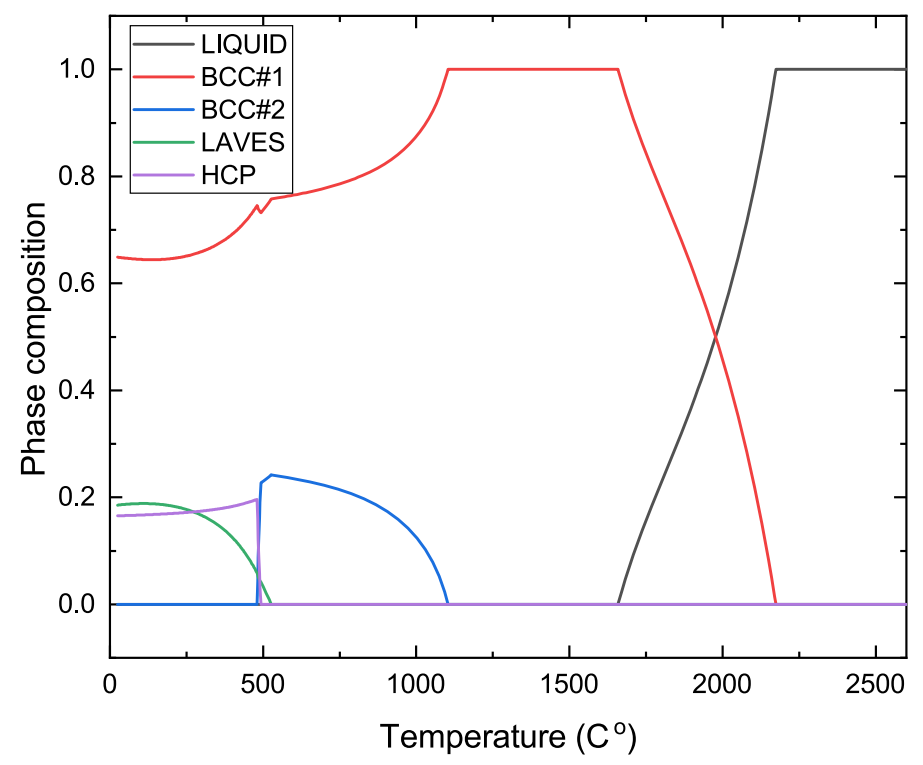

Fig. 2 Phase composition (normalized) of the HEA MoNbTaTiVZr, calculated via the CALPHAD method

\section{Results}

3.1 Thermodynamic calculation of phase compositions

The calculated (CALPHAD) normalized phase composition of MoNbTaTiVZr is plotted against the temperature in Fig. 2. While cooling the alloy from the liquid state, the solidification begins at $2174{ }^{\circ} \mathrm{C}$ and ends at $1658{ }^{\circ} \mathrm{C}$ at thermodynamic equilibrium, forming a single-phase BCC\#1. However, such a large freezing range might result in an enhanced heterogeneity in the solidified phases [26]. The single-phase composition $\mathrm{BCC \# 1}$ is stable until the alloy is cooled to $1103^{\circ} \mathrm{C}$. Here BCC\#1 starts to partially transform into another phase (BCC\#2). At low temperatures, a gradual formation of the Laves phase is predicted at $527^{\circ} \mathrm{C}$, while an $\mathrm{HCP}$ phase appears and a sharp drop of the phase fraction BCC\#2 is predicted simultaneously at $493{ }^{\circ} \mathrm{C}$.

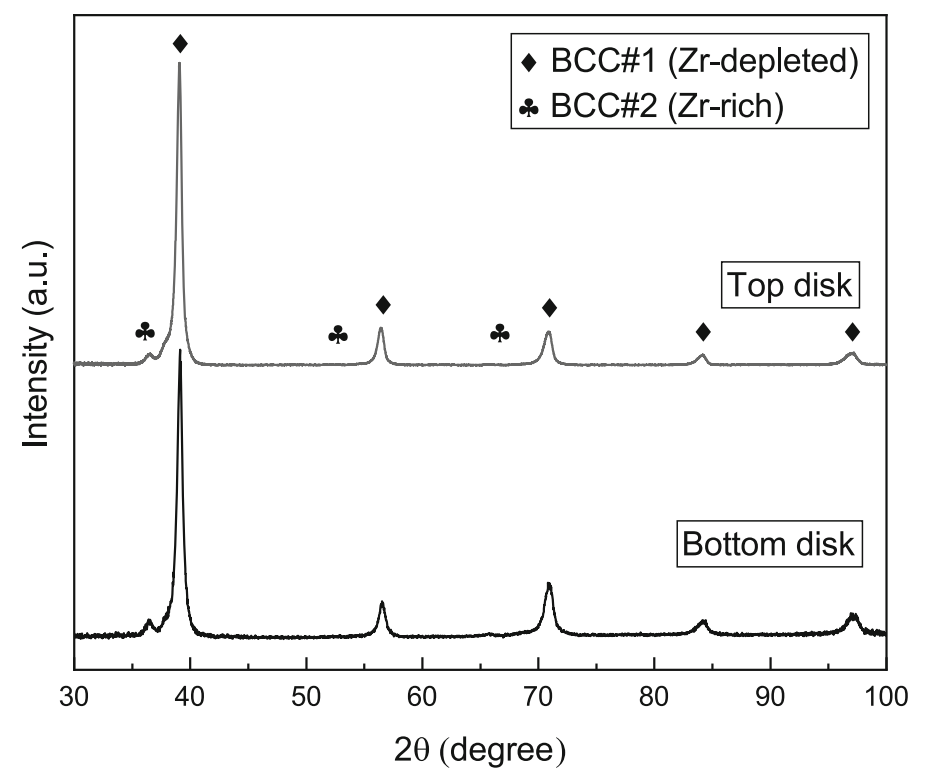

Fig. 3 X-ray diffraction (XRD) patterns of the top and bottom disks of the cylindrical specimens in the as-cast condition 

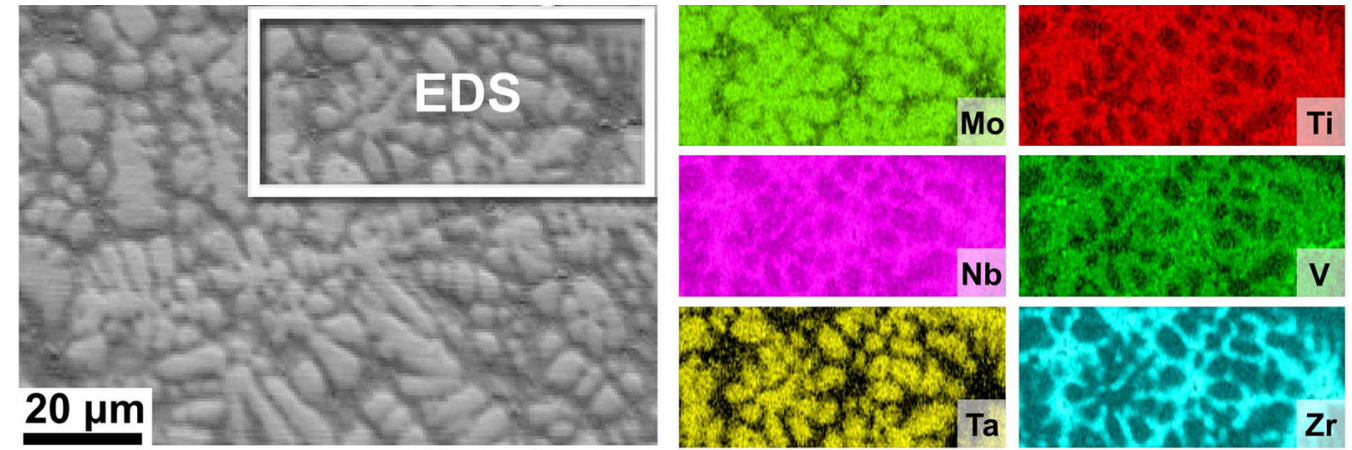

Fig. 4 Microstructure and the corresponding EDS mapping obtained in the as-cast condition

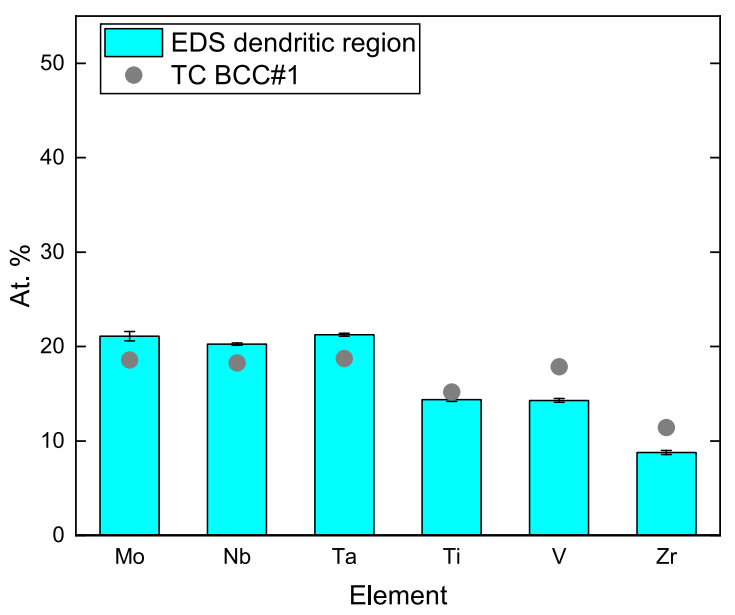

(a)

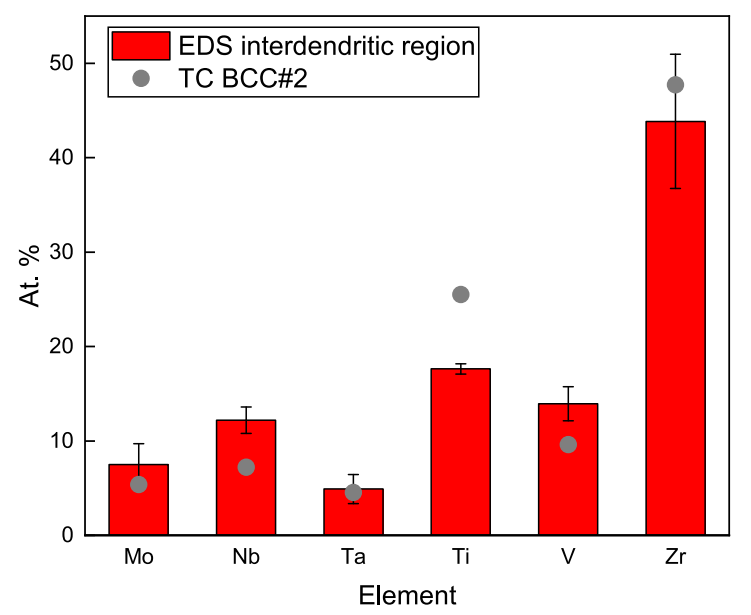

(b)

Fig. 5 Elemental concentration obtained from energy-dispersive X-ray spectroscopy (EDS) and thermodynamic calculations from ThermoCalc (TC) using the CALPHAD method

\subsection{Microstructural characterization of the as-cast HEA}

The XRD patterns of the disks before HPT shown in Fig. 3 indicate the existence of two BCC phases in both the top and bottom disks. There is no remarkable difference between the XRD patterns, which means that the disks sliced from the cylinder do not differ in their initial condition.

The secondary electron images of the alloy shown on the left side of Fig. 4 reveal a dendritic microstructure which consists of large dendritic and small interdendritic regions with a dendritic arm spacing of 10-15 $\mu \mathrm{m}$. The top right edge of the detection area is further analyzed by EDS mapping. The colored maps show a strong segregation of $\mathrm{Ta}$ in the dendritic region and a considerable enrichment of $\mathrm{Zr}$ in the interdendritic region. Furthermore, Mo and $\mathrm{Nb}$ are slightly enriched in the dendritic regions, whereas $\mathrm{Ti}$ and $\mathrm{V}$ are more likely to accumulate in the interdendritic regions. Using EDS point analysis, the elemental concentrations are quantified, and the results are shown as histograms in Fig. 5a (dendritic) and 5b (interdendritic), respectively. The results confirm the segregation of $\mathrm{Zr}$. Note that the interdendritic region is much smaller than the dendritic region, and the EDS gathers the volumetric information of the specimen. Hence, the EDS measurement of the interdendritic region may contain a certain amount of information from the dendritic region, which leads to relatively large error bars for the interdendritic regions compared to those from the dendritic regions. In the EDS point analysis, the enrichment of Ta is less evident than in the EDS mappings. This is probably due to the fact that the intensity in EDS mapping is given as mass percentage, while the values from point analysis are atomic percentages.

The full profile analysis of the HEXRD diffractogram based on Rietveld refinement using the software MAUD is illustrated in Fig. 6. It should be noted that the crystal structure files (CIF files) in the refinement 


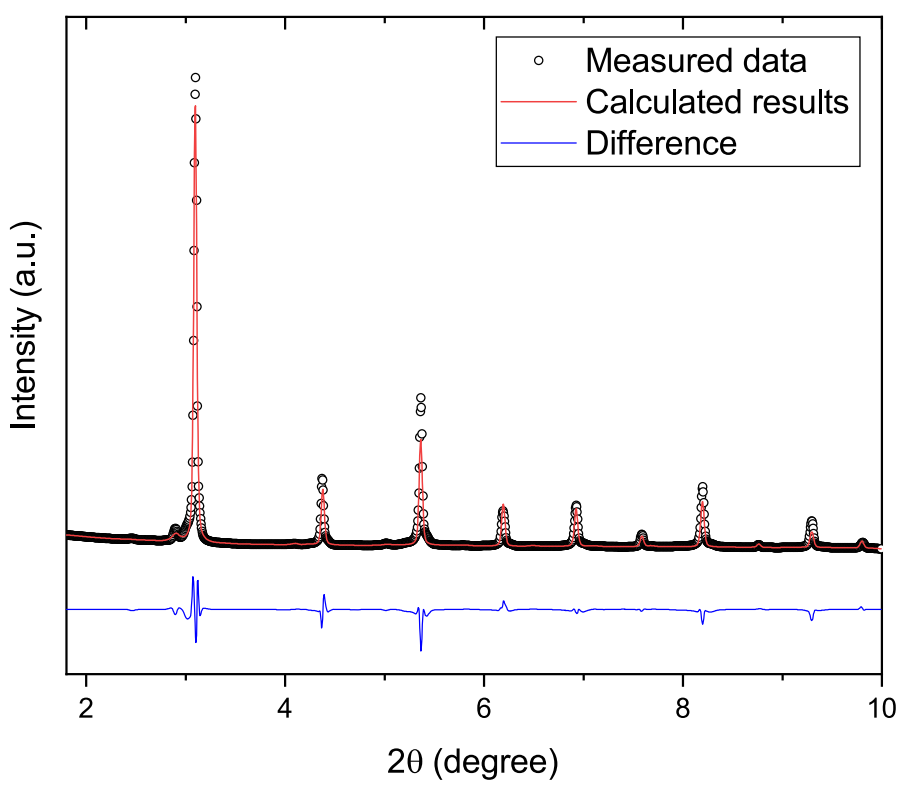

Fig. 6 High-energy X-ray diffraction (HEXRD) results and the full profile analysis using Rietveld refinement

Table 1 Results from Rietveld refinement of high-energy X-ray diffraction (HEXRD) and phase volume fraction from ThermoCalc (TC) at $1000^{\circ} \mathrm{C}$

\begin{tabular}{llll}
\hline Phase & $\mathrm{a}(\AA)$ & Vol. frac. (HEXRD) & Vol. frac. $\left(\mathrm{TC}, 1000{ }^{\circ} \mathrm{C}\right)$ \\
\hline BCC\#1 & 3.246 & $84 \%$ & $86 \%$ \\
BCC\#2 & 3.465 & $16 \%$ & $14 \%$ \\
\hline
\end{tabular}

are created according to EDS results and assume that both BCC solid solutions are disordered. Since the cylindrical specimen was measured in three directions only, the texture information is not sufficient and thus is not taken into consideration within the refinement. The results of the refinement are listed in Table 1. The volume fractions obtained from HEXRD are approximately in accordance with those from the thermodynamic calculation at $1000{ }^{\circ} \mathrm{C}$. This temperature was selected deliberately since the specimens are in the state of thermodynamic non-equilibrium due to the high cooling rate during production. This is evidenced by the fact that no Laves and HCP phases were observed in the diffractograms. In addition to that, the calculated elemental composition of each phase, as shown by the gray points in Fig. 5, corresponds almost exactly to the results obtained by EDS.

In Fig. 7, the image quality (IQ; a) map, color-coded EBSD orientation map (OM; b), high angle grain boundary (HAGB; c) map, BCC\#1 OM (d), BCC\#2 OM (e) and phase distribution map (f) of the as-cast alloy are shown. The grains are coarse with a size above $100 \mu \mathrm{m}$. The dendritic and interdendritic regions are identified as BCC\#1 and BCC\#2, respectively. These two BCC crystals are likely to co-exist in one grain.

Since BCC\#1 has a low concentration of $\mathrm{Zr}$ and BCC\#2 is enriched in $\mathrm{Zr}$, the two phases will henceforth be referred to as $\mathrm{Zr}$-depleted and $\mathrm{Zr}$-rich phases.

\subsection{Microstructural evolution during high-pressure torsion}

The equivalent shear strain during HPT can be calculated by

$$
\varepsilon=\frac{2 \pi N r}{\sqrt{3} t}, \quad 0 \leq r \leq R,
$$

where $N$ is the number of revolutions, $R$ is the radius of the disk and $t$ is its thickness [32].

Figure 8 shows XRD patterns of the disks processed by $0,1 / 4$ and 4 revolutions. The results reveal that no new phase is formed and the peaks of both phases are broadened. The peak of the $\mathrm{Zr}$-rich BCC\#2 phase 

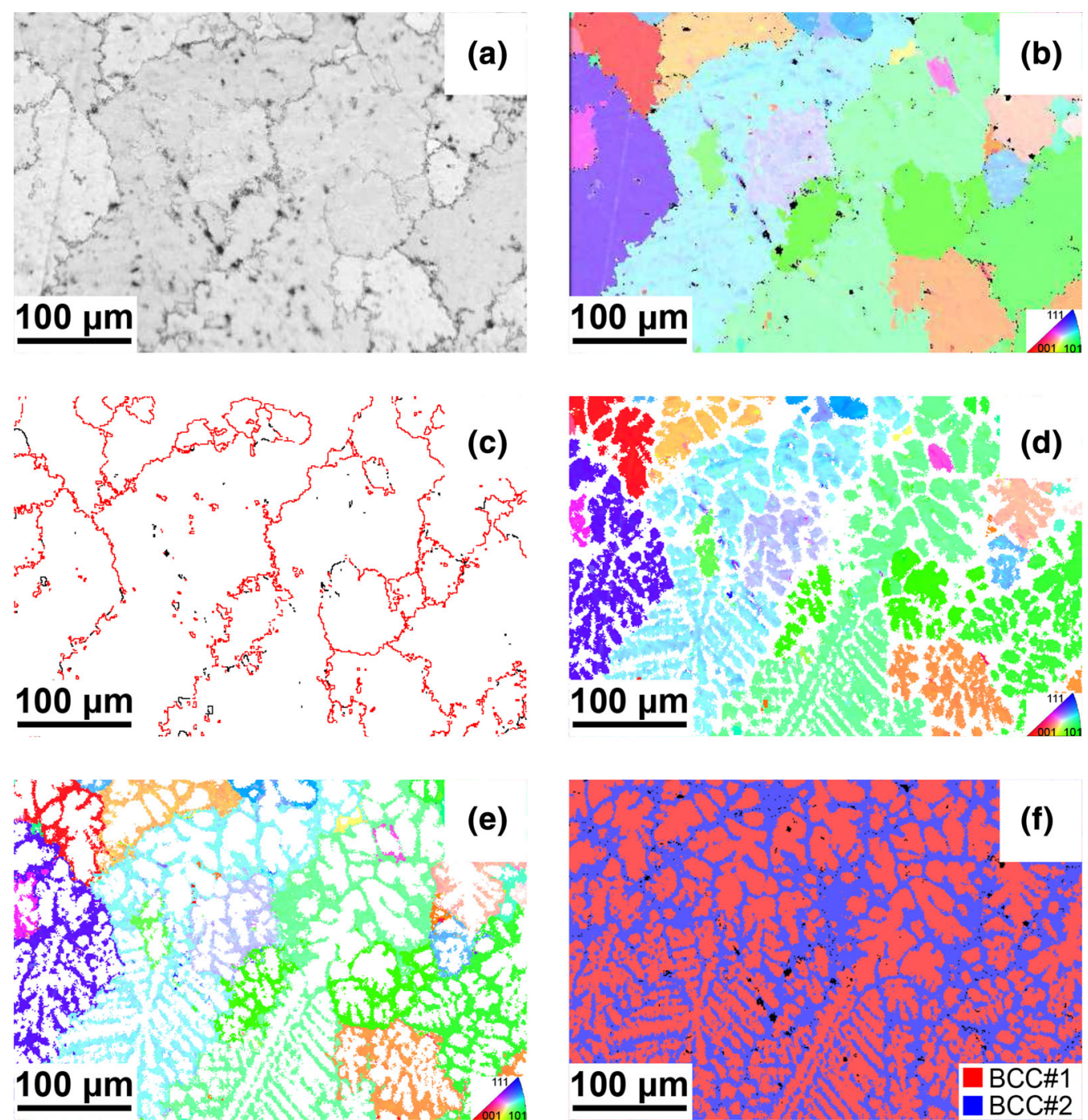

Fig. 7 Electron backscattered diffraction (EBSD) results of the as-cast HEA; (a) image quality (IQ) map, (b) color-coded EBSD orientation map (OM), (c) high angle grain boundary (HAGB) map, d BCC\#1 OM, (e) BCC\#2 OM and (f) phase distribution map

has a relatively weak intensity in the initial condition and almost disappears after four revolutions. The small peak to the right of the (110) peak in the $\mathrm{Zr}$-depleted phase (BCC\#1) originates from copper present in the embedding medium. Due to the small interaction volume and the difference in irradiated area resulting from angular change (especially at small angles), quantitative analyses of the dislocation density and grain size are not reliable. Further HEXRD measurements are planned to address these aspects.

Figure 9 shows microstructural observations of HPT-processed disks. The dendritic region can still be observed after $1 / 4$ revolution both near the center $(0.5 \mathrm{~mm}$ from the center) and at the periphery of the disk (2 $\mathrm{mm}$ from center). After two revolutions, the microstructure appears to be distorted at the periphery, showing a vortex-like structure, and small areas of the $\mathrm{Zr}$-rich phase can still be seen. After four revolutions, the two phases are well mixed. The $\mathrm{Zr}$-rich phase becomes remarkably elongated due to the shear strain introduced by HPT. The indents in Fig. 9 stem from hardness measurements, which will be the focus of sect. 3.4.

The EBSD orientation maps of the HPT-processed disks are shown in Fig. 10. The grain refinement with increasing shear strain can be clearly seen. Nevertheless, the dark areas, which are unresolved in EBSD identification and referred to as zero solutions, also increase simultaneously to grain refinement. A quantification of EBSD results is shown in Fig. 11. The percentage of zero solutions after 4 revolutions exceeds 98\%, i.e., 


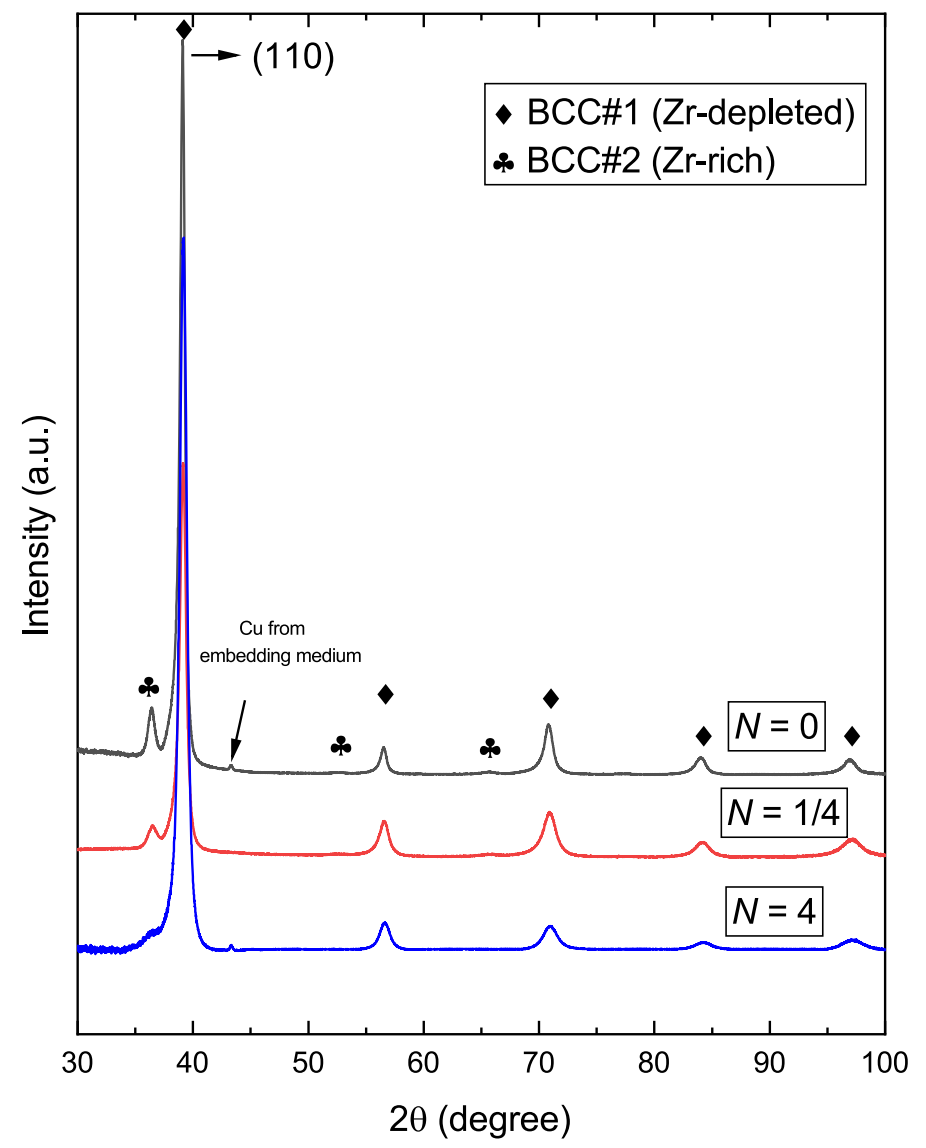

Fig. 8 X-ray diffraction (XRD) patterns obtained in axial direction at a radius of $2 \mathrm{~mm}$ of the disks after HPT, $N$ is the number of revolutions
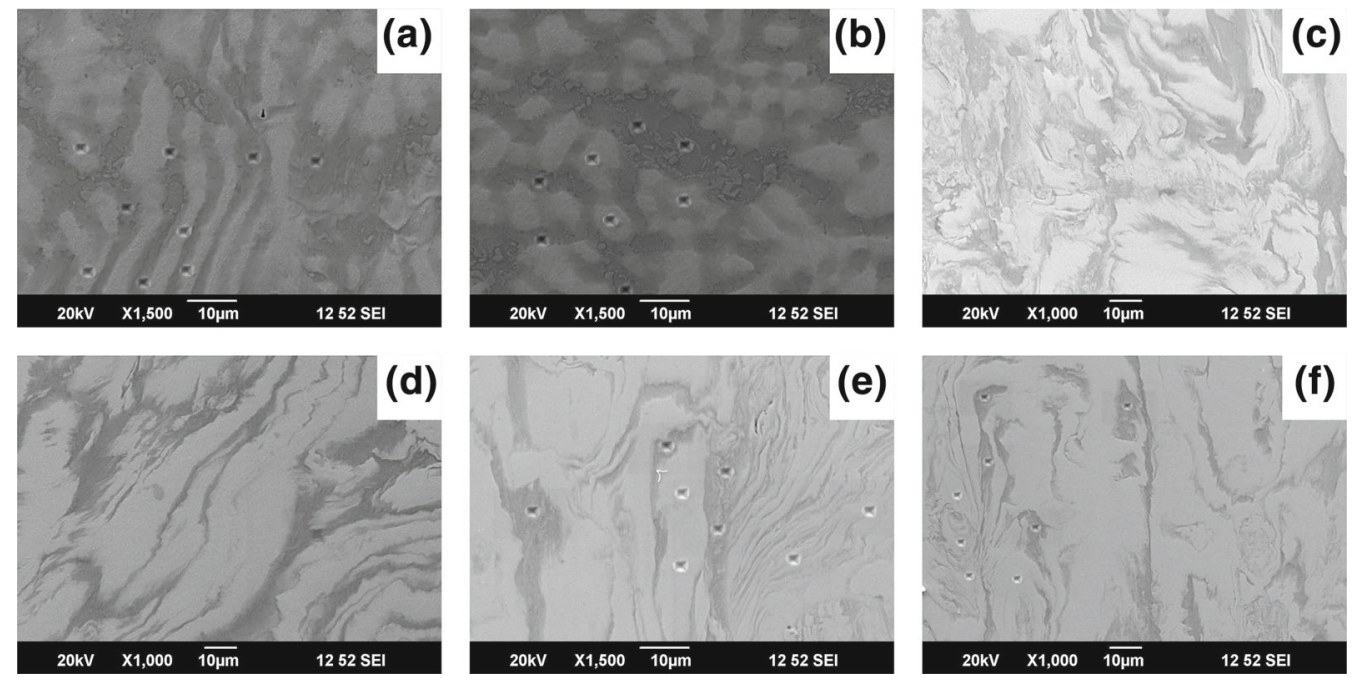

Fig. 9 Secondary electron micrographs in axial direction after HPT: (a) $N=1 / 4, r=0.5 \mathrm{~mm}$; (b) $N=1 / 4, r=2 \mathrm{~mm}$; (c) $N=$ $2, r=2 \mathrm{~mm}$ (d) $N=2, r=2.5 \mathrm{~mm}$; (e) $N=4, r=2 \mathrm{~mm}$ and (f) $N=4, r=2.5 \mathrm{~mm}$, where $N$ is the number of revolutions, $r$ is the distance from the center of the disks. The corresponding equivalent strains calculated from eq. (1) are (a) 0.76 , (b) 3.02, (c) 24.18, (d) 30.23 , (e) 48.37 and (f) 60.46 

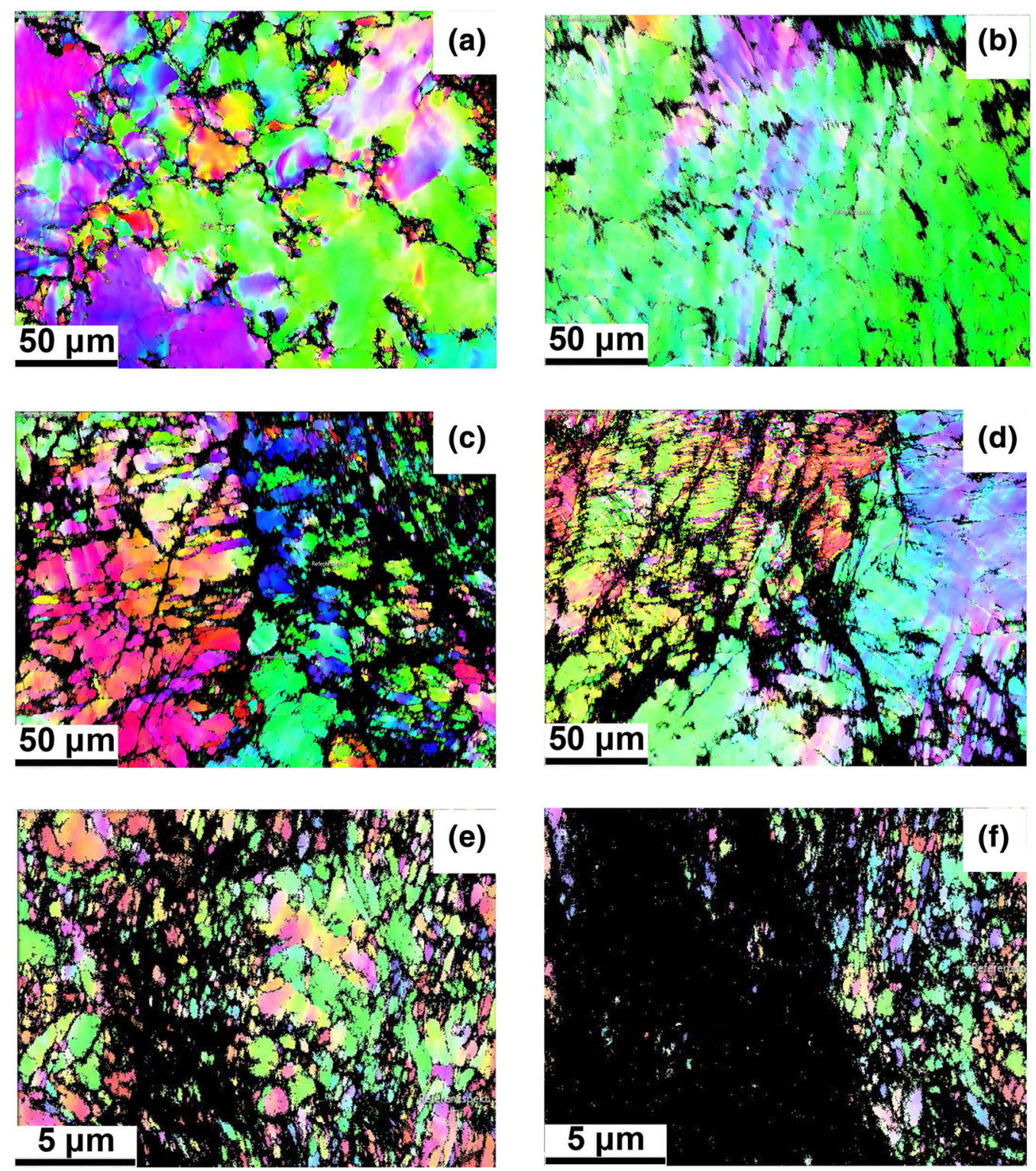

Fig. 10 EBSD orientation maps in axial direction of the disk (a) compressed without torsion at center; processed by $1 / 4$ revolution at (b) $1 \mathrm{~mm}$, (c) $2 \mathrm{~mm}$ and (d) $2.5 \mathrm{~mm}$ from the center; processed by two revolutions at (e) $1 \mathrm{~mm}$, (f) $2 \mathrm{~mm}$ from the center; the corresponding equivalent strains calculated from eq. (1) are (a) 0 , (b) 1.51 , (c) 3.02 , (d) 3.78 , (e) 12.09 and (f) 24.18

almost the whole detecting area remains unidentified. Thus, the results after four revolutions are not shown in Fig. 10.

The elemental concentrations in the disk after four revolutions along the radial direction are shown in Fig. 12a (Zr-depleted) and b (Zr-rich). The initial condition (as-cast) is marked in gray. Both BCC phases exhibit a tendency to homogenize and the concentration of all elements approach the ideal value of $16.67 \%$ for a 6-component HEA. In the $\mathrm{Zr}$-depleted phase there is a marked drop in the $\mathrm{Mo}, \mathrm{Nb}, \mathrm{Ta}$ and a notable increase in the Ti, $\mathrm{V}, \mathrm{Zr}$ concentrations. A reverse trend occurs in the $\mathrm{Zr}$-rich phase. The mean concentration of $\mathrm{Zr}$ in the $\mathrm{Zr}$-depleted phase increases for small distances from the center of the disk, while that in the $\mathrm{Zr}$-rich phase decreases only for distances larger than $1 \mathrm{~mm}$. However, large error bars of the Zr-concentration can be observed at the center and at small distances in the $\mathrm{Zr}$-rich phase indicating a large variation in the local chemical composition. Such a large variation of the chemical composition in the center region of the disk could be due to deviations from the ideal setup of the HPT, such as a misalignment of the axes of the anvils [33]. This could explain the observation that the concentrations of the elements vary in the enter of the disks even 


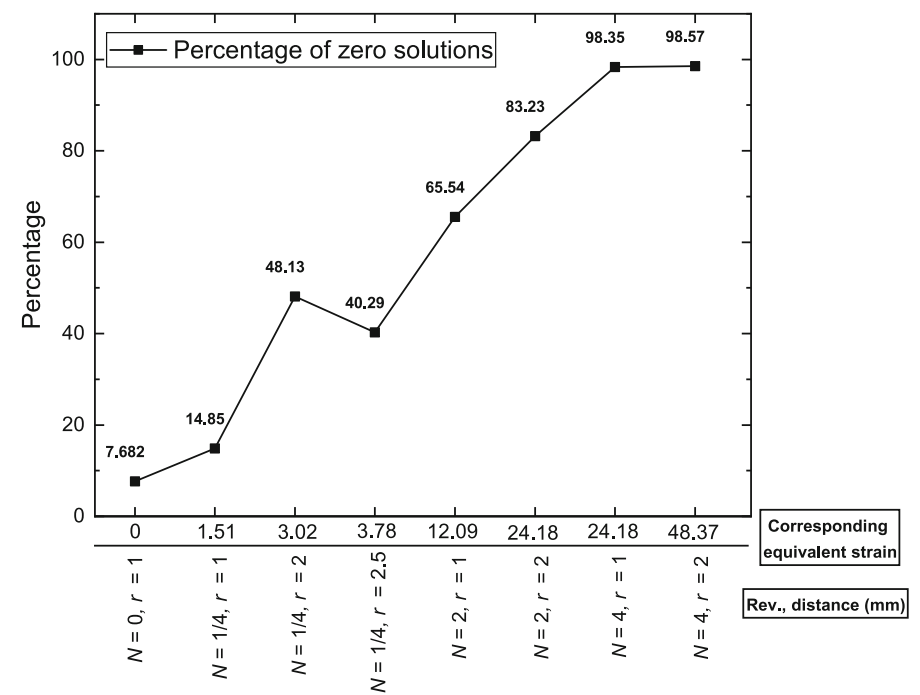

Fig. 11 Percentage of zero solutions (unresolved area) in the EBSD-analysis of HPT-processed disks. $r$ is the distance from the center of the disks, $N$ is the number of revolutions. The corresponding strains are calculated from eq. (1)

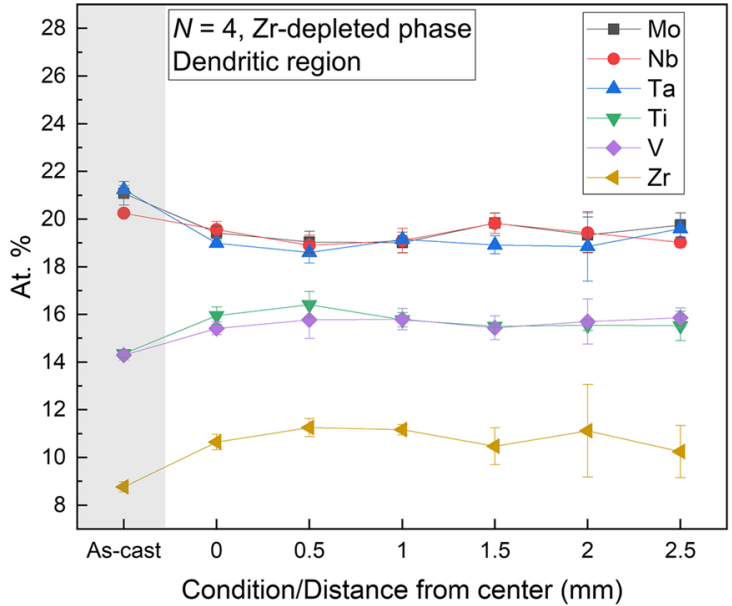

(a)

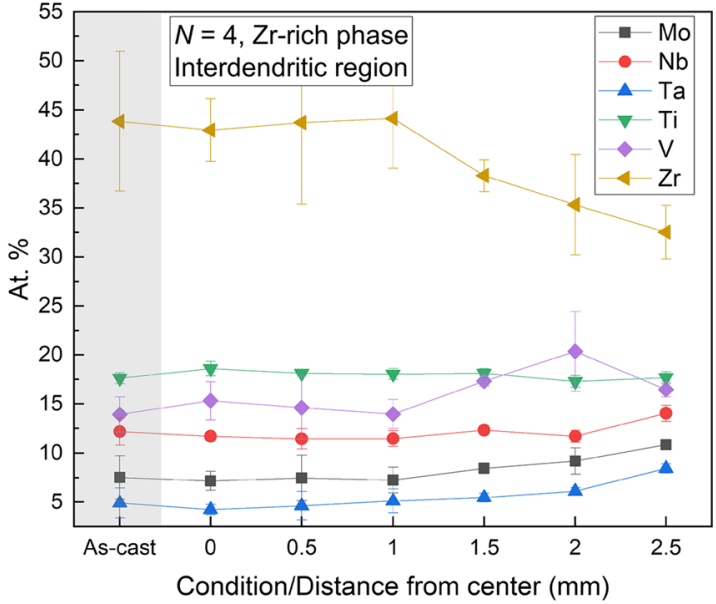

(b)

Fig. 12 Chemical composition obtained in axial direction of (a) the Zr-depleted and (b) the Zr-rich phase after four revolutions along the radial direction in comparison to the initial (as-cast) condition

after 4 revolutions. The mean concentration of $\mathrm{Zr}$ in the $\mathrm{Zr}$-rich phase shows a larger variation $\left(\Delta C_{\mathrm{Zr}_{\text {rich }}} \approx\right.$ $11.5 \%)$ than that of other elements in both phases $\left(\Delta C_{\mathrm{x}} \approx 5 \%\right)$.

\subsection{Hardness evolution during HPT and strain-hardening properties}

The ultra-micro-Vickers hardness of the Zr-depleted and Zr-rich phases along the diameter of HPT-processed disks are presented in Fig. 13a and b, respectively. For comparison, the hardness of both phases before HPT and also the error bars are marked in gray. While the Zr-depleted phase hardens, the Zr-rich phase seems to soften.

Hardness is plotted against the equivalent strain in Fig. 14a and $\mathrm{b}$. The variation of hardness in the sample with $1 / 4$ revolution is more pronounced than in the disks with two and four revolutions. A saturation of softening can be observed in the Zr-rich phase, while this phenomenon is not obvious for the hardening of the Zr-depleted phase. 


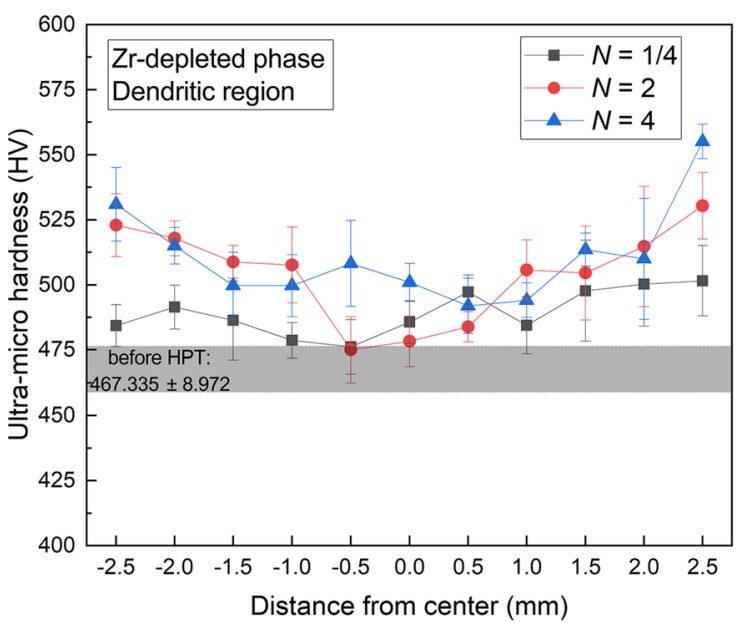

(a)

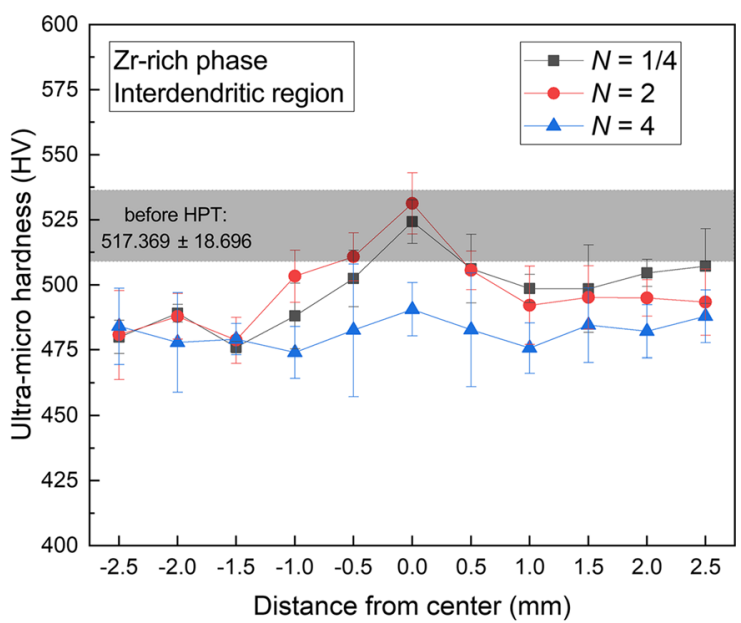

(b)

Fig. 13 Ultra-microhardness evolution in (a) the Zr-depleted and (b) the Zr-rich phase, obtained in axial direction, $N$ is the number of revolutions

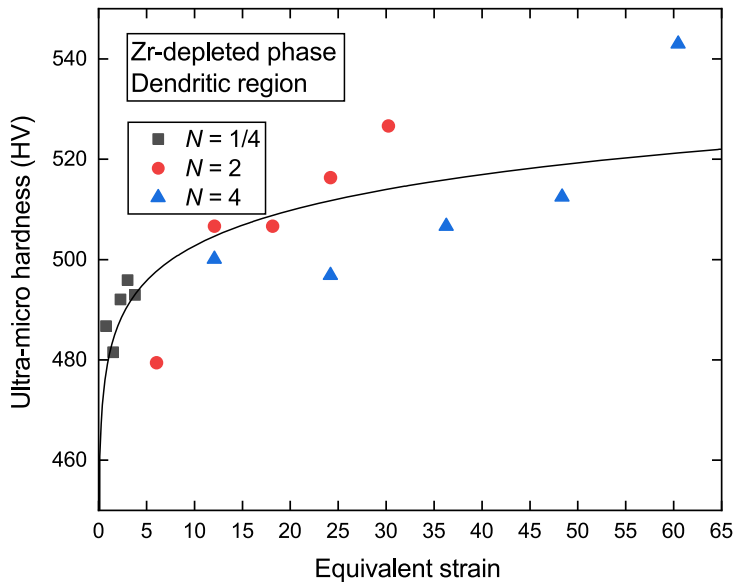

(a)

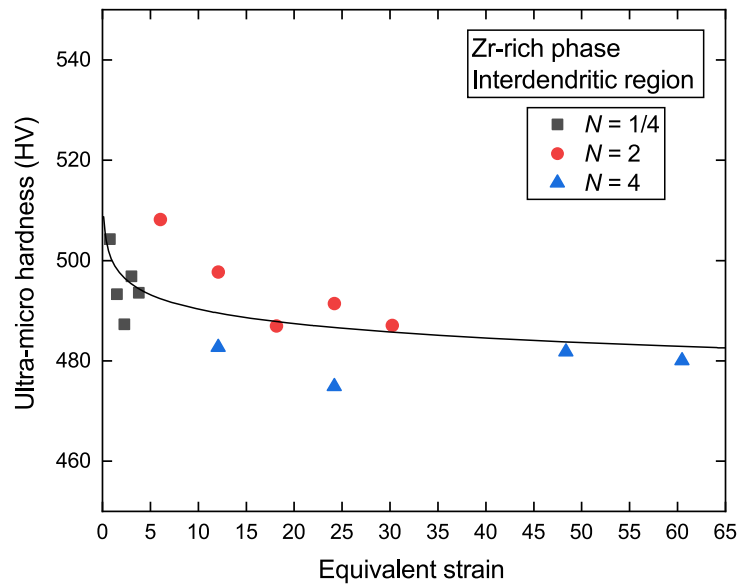

(b)

Fig. 14 Ultra-microhardness of (a) the Zr-depleted and (b) the Zr-rich phase as the function of equivalent strain, obtained in axial direction, $N$ is the number of revolutions

Figure 15 shows that the logarithm of hardness HV depends linearly on the logarithm of the equivalent strain $\varepsilon$. Hence, hardness depends on the equivalent strain similarly to [34] as:

$$
\begin{aligned}
\mathrm{HV}_{\mathrm{Zr}_{\text {depleted }}} & =480 \varepsilon^{0.0201}, \\
\mathrm{HV}_{\mathrm{Zr}_{\text {rich }}} & =500 \varepsilon^{-0.0085} .
\end{aligned}
$$

\section{Discussion}

The HEA studied in this work exhibits a dual-phase crystal structure consisting of two BCC phases (Fig. 3) and dendritic microstructure (Fig. 7).

The results of local hardness measurements (Fig. 13) clearly show that the two BCC phases possess an opposite strain-hardening behavior. The hardness of the $\mathrm{Zr}$-depleted phase increases to $\sim 540 \mathrm{HV}$ at the periphery of the disk after four revolutions, while that of the Zr-rich phase decreases to $\sim 480 \mathrm{HV}$. Similar results on the saturation hardness in single-phase BCC HEAs were reported in [23,24,35].

Softening during HPT could be attributed to static and dynamic recrystallization [36,37], grain boundaries acting as dislocation sinks [38], or the decomposition of supersaturated solid solutions [39]. An inspection 
Table 2 Atomic radii of the component elements in the HEA studied in this work [40]

\begin{tabular}{lllllll}
\hline & Mo & $\mathrm{Nb}$ & $\mathrm{Ta}$ & $\mathrm{Ti}$ & $\mathrm{V}$ & $\mathrm{Zr}$ \\
\hline Atomic radii $(\AA)$ & 1.40 & 1.47 & 1.47 & 1.46 & 1.35 & 1.60 \\
\hline
\end{tabular}

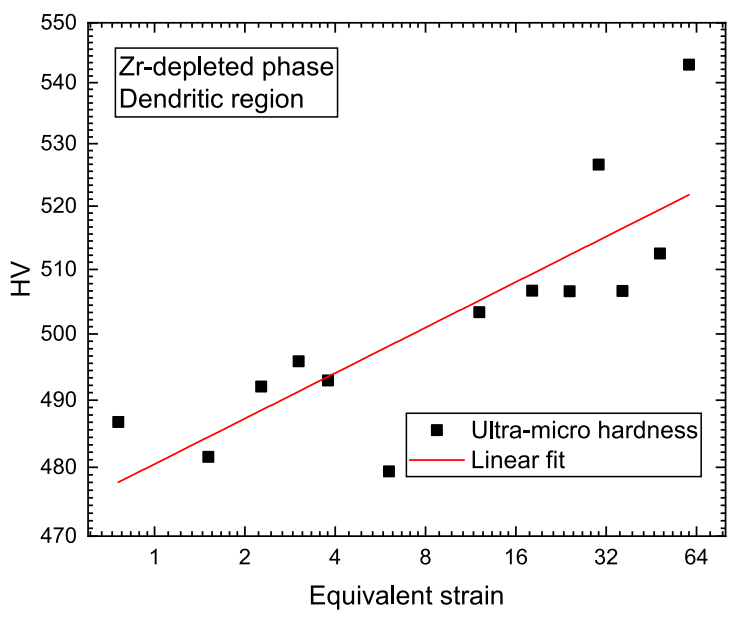

(a)

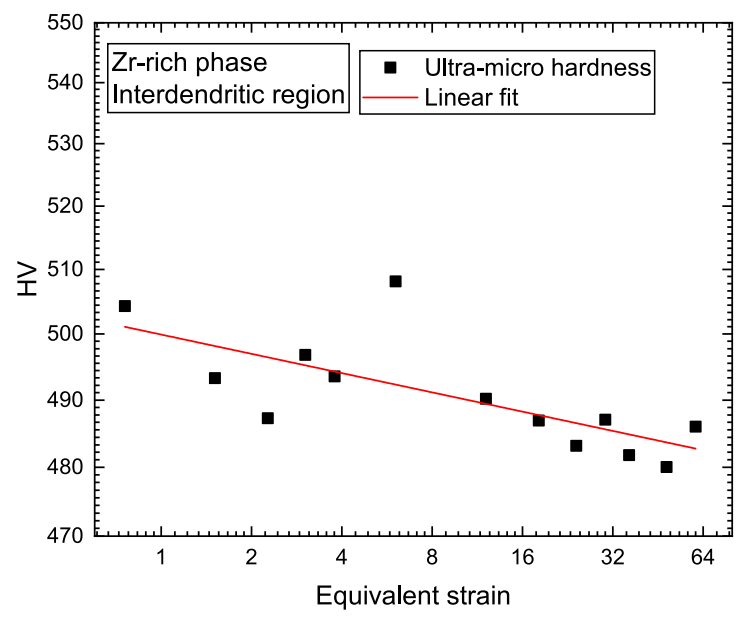

(b)

Fig. 15 Ultra-microhardness of (a) the Zr-depleted and (b) the Zr-rich phase plotted against the equivalent strain (log-log-plot), obtained in axial direction

of the EDS results showing a decrease in the $\mathrm{Zr}$ concentration may hint to the last hypothesis. Solid solution strengthening arises from lattice misfit due to the difference in atomic radii between the solute and the solvent atoms. As shown in Table 2, Zr possesses the largest atomic radius among the six elements of our HEA [40], which indicates that $\mathrm{Zr}$ might play a prominent role in this alloy. A decrease in the concentration of $\mathrm{Zr}$ may diminish this hardening effect and could lead to softening. If this softening exceeds hardening effects such as grain refinement and an increase in dislocation density, the overall result would be softening. Meanwhile, the solid solution hardening of the $\mathrm{Zr}$-depleted phase would be enhanced thus resulting in an overall strengthening of this phase. However, this analysis provides only a rough estimate, as a careful examination would require a pair distribution function analysis [41].

Since the samples were processed at RT with extremely low rotational speed (0.2 RPM), and the diffusion of atoms in HEAs is reported to be sluggish [1], the homogenization of the alloy should be overwhelmingly driven by the shear strains under high-pressure instead of being driven by thermal activation. Similar to the microstructural observations made in this work (Fig. 9d and f), recent studies on shear-induced mixing and co-deformation of dissimilar materials and heterophases [13-15] also reported vortex-like microstructures. It was suggested $[13,14]$ that such microstructure resulted from a shear-induced mutual diffusive mass transfer across the interface between the constituents, analogous to turbulence in fluid dynamics.

The irregularity and the large error bars observed in Fig. 13 might be attributed to strain localization, which could be related to the plastic mismatch of constituents in HPT-processed dual-phase materials [42]. In our case, where the constituents show little difference in hardness, the localization might be associated with the non-planar core structure of screw dislocations in BCC crystals that hinders dislocation motion [43]. Upon plastic deformation, the dislocations could interact with their non-planar core, leading to critical resolved shear stresses that are at least an order of magnitude larger than in the case of dislocations possessing a planar core [44]. The dislocation motion is not only influenced by the shear stress parallel to the Burgers vector but also by other stress components $[43,44]$. This might result in a complex deformation behavior and local heterogeneity in materials with BCC structure $[43,44]$. Such a heterogeneity could be further enhanced by the existence of the interfaces between the Zr-depleted and the Zr-rich phases (see Fig. 7b, d and e). Such interfaces could impede dislocation motion.

After two revolutions, the grain size was refined from over $100 \mu \mathrm{m}$ in the initial condition (Fig. 7b) to $500 \mathrm{~nm}$ in the Zr-depleted phase and $\sim 100 \mathrm{~nm}$ in the Zr-rich phase (Fig. 10f). As reported by Schuh et al. [24], the grain size of a single-phase BCC HEA (HfNbTaTiZr) during HPT could be refined to $\sim 50 \mathrm{~nm}$ in the saturated condition. This discrepancy of grain size reported in this work is most likely due to the limitations of 
employing EBSD for grain size determination of severely plastically deformed crystals. As mentioned above, a large plastic strain leads to a high amount of unresolved sample areas as was also reported in [45]. To gain more reliable grain size values needed to quantify the relationship between grain size and straining, transmission electron microscopy should be employed for this purpose.

\section{Conclusions}

In the present work, the strain-hardening properties of a dual-phase refractory HEA with equimolar composition of $\mathrm{Mo}, \mathrm{Nb}, \mathrm{Ta}, \mathrm{Ti}, \mathrm{V}$ and $\mathrm{Zr}$ were studied. The following conclusions can be drawn.

1. CALPHAD results reveal the occurrence of two BCC, a Laves and an HCP phase in equilibrium. However, in the as-cast condition, the phase composition predicted by thermodynamic calculations conducted at $1000^{\circ} \mathrm{C}$ and microstructure characterizations indicate the existence of a dual-phase structure consisting of about $85 \% \mathrm{Zr}$-depleted BCC and $15 \% \mathrm{Zr}$-rich BCC phase.

2. After two revolutions, HPT processing leads to a grain refinement to several hundred nanometers. The grain size after four revolutions cannot be determined by EBSD, since the percentage of zero solutions exceeds $98 \%$.

3. The two BCC phases exhibit opposite hardening behavior. The softening of the $\mathrm{Zr}$-rich phase seems to be correlated with the shear-induced atomic transport of $\mathrm{Zr}$, which is enhanced by the formation of a vortexlike microstructure. The high scatter of the hardness values may be attributed to strain localization during HPT.

Further investigations on the hardening effect due to higher numbers of revolutions in HPT are necessary, as the saturation hardness plateau is not evident in the $\mathrm{Zr}$-depleted phase. In addition, other techniques than EBSD should be employed for grain size determination.

Acknowledgements The authors would like to thank DESY (Hamburg, Germany) for support and access to HEXRD facilities. Part of the experiments was conducted at PETRA III. Moreover, part of this work is based upon samples prepared at the Materials Science Lab operated by FRM II and Helmholtz-Zentrum Hereon at the Heinz Maier-Leibnitz Zentrum (MLZ), Garching, Germany, which is gratefully acknowledged.

Funding Open Access funding enabled and organized by Projekt DEAL.

Open Access This article is licensed under a Creative Commons Attribution 4.0 International License, which permits use, sharing, adaptation, distribution and reproduction in any medium or format, as long as you give appropriate credit to the original author(s) and the source, provide a link to the Creative Commons licence, and indicate if changes were made. The images or other third party material in this article are included in the article's Creative Commons licence, unless indicated otherwise in a credit line to the material. If material is not included in the article's Creative Commons licence and your intended use is not permitted by statutory regulation or exceeds the permitted use, you will need to obtain permission directly from the copyright holder. To view a copy of this licence, visit http://creativecommons.org/licenses/by/4.0/.

\section{References}

1. Miracle, D.B., Senkov, O.N.: A critical review of high entropy alloys and related concepts. Acta Mater. 122, 448-511 (2017)

2. Murty, B.S., Yeh, J.W., Ranganathan, S., Bhattacharjee, P.P.: High-Entropy Alloys. Elsevier, Amsterdam (2019)

3. Senkov, O.N., Wilks, G.B., Miracle, D.B., Chuang, C.P., Liaw, P.K.: Refractory high-entropy alloys. Intermetallics 18(9), $1758-1765(2010)$

4. Zhang, Y., Zuo, T.T., Tang, Z., Gao, M.C., Dahmen, K.A., Liaw, P.K., Lu, Z.P.: Microstructures and properties of high-entropy alloys. Prog. Mater Sci. 61, 1-93 (2014)

5. Yeh, J.W., Chen, S.K., Lin, S.J., Gan, J.Y., Chin, T.S., Shun, T.T., Tsau, C.H., Chang, S.Y.: Nanostructured high-entropy alloys with multiple principal elements: novel alloy design concepts and outcomes. Adv. Eng. Mater. 6(5), 299-303 (2004)

6. Reiberg, M., von Kobylinski, J., Werner, E.: Characterization of powder metallurgically produced AlCrFeNiTi multi-principle element alloys. Continuum Mech. Thermodyn. 32(4), 1147-1158 (2020)

7. Reiberg, M., Li, X., Maawad, E., Werner, E.: Lattice strain during compressive loading of AlCrFeNiTi multi-principal element alloys. Continuum Mech. Thermodyn. 33, 1541-1554 (2021)

8. Senkov, O.N., Gorsse, S., Miracle, D.B.: High temperature strength of refractory complex concentrated alloys. Acta Mater. 175, 394-405 (2019)

9. Senkov, O.N., Miracle, D.B., Chaput, K.J., Couzinie, J.P.: Development and exploration of refractory high entropy alloys-a review. J. Mater. Res. 33(19), 3092-3128 (2018)

10. Senkov, O.N., Senkova, S.V., Miracle, D.B., Woodward, C.: Mechanical properties of low-density, refractory multi-principal element alloys of the Cr-Nb-Ti-V-Zr system. Mater. Sci. Eng. A 565, 51-62 (2013) 
11. Pippan, R., Scheriau, S., Taylor, A., Hafok, M., Hohenwarter, A., Bachmaier, A.: Saturation of fragmentation during severe plastic deformation. Annu. Rev. Mater. Res. 40(1), 319-343 (2010)

12. Zhilyaev, A.P., Langdon, T.G.: Using high-pressure torsion for metal processing: fundamentals and applications. Prog. Mater Sci. 53(6), 893-979 (2008)

13. Kulagin, R., Beygelzimer, Y., Ivanisenko, Y., Mazilkin, A., Straumal, B., Hahn, H.: Instabilities of interfaces between dissimilar metals induced by high pressure torsion. Mater. Lett. 222, 172-175 (2018)

14. Pouryazdan, M., Kaus, B.J., Rack, A., Ershov, A., Hahn, H.: Mixing instabilities during shearing of metals. Nat. Commun. 8(1), 1-7 (2017)

15. Taheriniya, S., Davani, F.A., Hilke, S., Hepp, M., Gadelmeier, C., Chellali, M.R., Boll, T., Rösner, H., Peterlechner, M., Gammer, C., Divinski, S.V., Butz, B., Glatzel, U., Hahn, H., Wilde, G.: High entropy alloy nanocomposites produced by high pressure torsion. Acta Materialia 208,(2021)

16. Guo, W., Jägle, E.A., Choi, P.P., Yao, J., Kostka, A., Schneider, J.M., Raabe, D.: Shear-induced mixing governs codeformation of crystalline-amorphous nanolaminates. Phys. Rev. Lett. 113(3), 1-5 (2014)

17. Straumal, B.B., Pontikis, V., Kilmametov, A.R., Mazilkin, A.A., Dobatkin, S.V., Baretzky, B.: Competition between precipitation and dissolution in $\mathrm{Cu}-\mathrm{Ag}$ alloys under high pressure torsion. Acta Mater. 122, 60-71 (2017)

18. Setman, D., Schafler, E., Korznikova, E., Zehetbauer, M.J.: The presence and nature of vacancy type defects in nanometals detained by severe plastic deformation. Mater. Sci. Eng., A 493(1-2), 116-122 (2008)

19. Moon, J., Qi, Y., Tabachnikova, E., Estrin, Y., Choi, W.M., Joo, S.H., Lee, B.J., Podolskiy, A., Tikhonovsky, M., Kim, H.S.: Microstructure and mechanical properties of high-entropy alloy Co20Cr26Fe20Mn20Ni14 processed by high-pressure torsion at $77 \mathrm{~K}$ and $300 \mathrm{~K}$. Sci. Rep. 8(1), 1-12 (2018)

20. Praveen, S., Bae, J.W., Asghari-Rad, P., Park, J.M., Kim, H.S.: Ultra-high tensile strength nanocrystalline CoCrNi equi-atomic medium entropy alloy processed by high-pressure torsion. Mater. Sci. Eng., A 735, 394-397 (2018)

21. Schuh, B., Mendez-Martin, F., Völker, B., George, E.P., Clemens, H., Pippan, R., Hohenwarter, A.: Mechanical properties, microstructure and thermal stability of a nanocrystalline $\mathrm{CoCrFeMnNi}$ high-entropy alloy after severe plastic deformation. Acta Mater. 96, 258-268 (2015)

22. Zheng, R., Chen, J., Xiao, W., Ma, C.: Microstructure and tensile properties of nanocrystalline (FeNiCoCu) $1-x \operatorname{Ti} x \mathrm{Al} x$ high entropy alloys processed by high pressure torsion. Intermetallics 74, 38-45 (2016)

23. Gubicza, J., Heczel, A., Kawasaki, M., Han, J.K., Zhao, Y., Xue, Y., Huang, S., Lábár, J.L.: Evolution of microstructure and hardness in Hf25Nb25Ti25Zr25 high-entropy alloy during high-pressure torsion. J. Alloy. Compd. 788, 318-328 (2019)

24. Schuh, B., Völker, B., Todt, J., Schell, N., Perrière, L., Li, J., Couzinié, J.P., Hohenwarter, A.: Thermodynamic instability of a nanocrystalline, single-phase TiZrNbHfTa alloy and its impact on the mechanical properties. Acta Mater. 142, 201-212 (2018)

25. Tang, Q.H., Huang, Y., Huang, Y.Y., Liao, X.Z., Langdon, T.G., Dai, P.Q.: Hardening of an Al0.3CoCrFeNi high entropy alloy via high-pressure torsion and thermal annealing. Mater. Lett. 151, 126-129 (2015)

26. Edalati, P., Floriano, R., Tang, Y., Mohammadi, A., Pereira, K.D., Luchessi, A.D., Edalati, K.: Ultrahigh hardness and biocompatibility of high-entropy alloy TiAlFeCoNi processed by high-pressure torsion. Mater. Sci. Eng., C 112, 110908 (2020)

27. Bachmaier, A., Pippan, R.: High-pressure torsion deformation induced phase transformations and formations: new material combinations and advanced properties. Mater. Trans. 60(7), 1256-1269 (2019)

28. Kormout, K.S., Pippan, R., Bachmaier, A.: Deformation-induced supersaturation in immiscible material systems during high-pressure torsion. Adv. Eng. Mater. 19(4), 1-19 (2017)

29. Stückler, M., Krenn, H., Kürnsteiner, P., Gault, B., De Geuser, F., Weissitsch, L., Wurster, S., Pippan, R., Bachmaier, A.: Intermixing of $\mathrm{Fe}$ and $\mathrm{Cu}$ on the atomic scale by high-pressure torsion as revealed by DC- and AC-SQUID susceptometry and atom probe tomography. Acta Mater. 196, 210-219 (2020)

30. Lutterotti, L., Matthies, S., Wenk, H.R.: MAUD (material analysis using diffraction): a user friendly Java program for Rietveld texture analysis and more. In: Proceeding of the twelfth international conference on textures of materials (ICOTOM-12), vol. 1, p. 1599. NRC Research Press Ottowa, Canada (1999)

31. Schneider, C.A., Rasband, W.S., Eliceiri, K.W.: NIH Image to ImageJ: 25 years of image analysis. Nat. Methods 9(7), 671-675 (2012)

32. Hebesberger, T., Stüwe, H.P., Vorhauer, A., Wetscher, F., Pippan, R.: Structure of $\mathrm{Cu}$ deformed by high pressure torsion. Acta Mater. 53(2), 393-402 (2005)

33. Vorhauer, A., Pippan, R.: On the homogeneity of deformation by high pressure torsion. Scripta Mater. 51(9), 921-925 (2004)

34. Wang, Y.C., Langdon, T.G.: Effect of heat treatment on microstructure and microhardness evolution in a Ti-6Al-4V alloy processed by high-pressure torsion. J. Mater. Sci. 48(13), 4646-4652 (2013)

35. Č́ížek, J., Haušild, P., Cieslar, M., Melikhova, O., Vlasák, T., Janeček, M., Král, R., Harcuba, P., Lukáč, F., Zýka, J., Málek, J., Moon, J., Kim, H.S.: Strength enhancement of high entropy alloy HfNbTaTiZr by severe plastic deformation. J. Alloy. Compd. 768, 924-937 (2018)

36. Edalati, K., Ito, Y., Suehiro, K., Horita, Z.: Softening of high purity aluminum and copper processed by high pressure torsion. Int. J. Mater. Res. 100(12), 1668-1673 (2009)

37. Xu, J., Li, J., Wang, C.T., Shan, D., Guo, B., Langdon, T.G.: Evidence for an early softening behavior in pure copper processed by high-pressure torsion. J. Mater. Sci. 51(4), 1923-1930 (2016)

38. Ito, Y., Edalati, K., Horita, Z.: High-pressure torsion of aluminum with ultrahigh purity (99.9999\%) and occurrence of inverse Hall-Petch relationship. Mater. Sci. Eng., A 679, 428-434 (2017)

39. Mazilkin, A., Straumal, B., Borodachenkova, M., Valiev, R., Kogtenkova, O., Baretzky, B.: Gradual softening of Al-Zn alloys during high-pressure torsion. Mater. Lett. 84, 63-65 (2012)

40. Kittel, C., McEuen, P., McEuen, P.: Introduction to solid state physics, vol. 8. Wiley, New York (1996)

41. Owen, L.R., Pickering, E.J., Playford, H.Y., Stone, H.J., Tucker, M.G., Jones, N.G.: An assessment of the lattice strain in the CrMnFeCoNi high-entropy alloy. Acta Mater. 122, 11-18 (2017) 
42. Sabirov, I., Pippan, R.: Formation of a W-25\%Cu nanocomposite during high pressure torsion. Scripta Mater. 52(12), 1293$1298(2005)$

43. Hirth, J.P., Lothe, J., Mura, T.: Theory of dislocations. J. Appl. Mech. 50(2), 476 (1983)

44. Vitek, V.: Dislocation Cores and Unconventional Properties of Plastic Behavior. Handbook of Materials Modeling pp. 2883$2896(2005)$

45. Zherebtsov, S., Stepanov, N., Ivanisenko, Y., Shaysultanov, D., Yurchenko, N., Klimova, M., Salishchev, G.: Evolution of microstructure and mechanical properties of a CoCrFeMnNi high-entropy alloy during high-pressure torsion at room and cryogenic temperatures. Metals 8(2),(2018)

Publisher's Note Springer Nature remains neutral with regard to jurisdictional claims in published maps and institutional affiliations. 